MedieKultur | Journal of media and communication research | ISSN 1901-9726

Article

\title{
Genre, technology and embodied interaction: The evolution of digital game genres and motion gaming
}

\section{Andreas Gregersen}

MedieKultur 2011, 51, 94-109

Published by SMID | Society of Media researchers In Denmark | www.smid.dk The online version of this text can be found open access at www.mediekultur.dk

Technology has been given relatively little attention in genre theory, but this article argues that material technologies can be important components in genre development. The argument is based on a historically informed analysis of digital games, with special attention paid to home console video games and recent genre developments within this domain commonly referred to as motion gaming.

The main point is that digital game genres imply structured embodied activity. A constitutive element of digital game mediation is a control interface geared to player embodiment, and I propose the concept of 'interaction modes' to describe the coupling of technology and player embodiment and show how this can be integrated with genre theory. The resulting framework allows for increased attention to continuity and change in game and communication genres, material and digital technologies, and the related interaction modes.

\section{Introduction}

When celebrated game designer Shigeru Miyamoto came on stage to introduce the Wii, i.e., Nintendo's new video game console, at the Electronic Entertainment Expo in 2006, his words were "Tennis, anyone? Controls are simple, even your mom can play." He held up a small, white stick-like object and proceeded to control a simple tennis game by swinging his 
arms and hands vigorously while moving around on stage. The Wii and its commercial success spearheaded a development within digital games now referred to as motion gaming by the gaming industry, journalists and bloggers. In this article, this term is used to identify and discuss a broader trend in gaming towards the physical movement of players and the related technologies involved. Importantly, Miyamoto did not just introduce a new console, but also a new controller technology, i.e., the Wiimote.

This article uses motion gaming as an entry point to a larger discussion of the roles played by material technology and player embodiment in genre developments within digital games. Genre theory in literary theory, film and television studies, and rhetoric has much to offer the scholar of digital media and digital games, but the role of material technologies tends to be underdeveloped in these writings, and this is where I aim to augment the existing genre theory frameworks. The article seeks both to track genre evolution and to develop the concept of genre with regards to digital games. In doing so, a tension between generality and specificity must be observed: by abstracting away from technology one gains the power of broad application, but loses the ability to see where material technologies, in casu controllers, have made important differences. In addition, technologies may take generic or highly specific forms.

My argument is represented by three parts. First, existing genre theories, as useful as they are, do not allow us to grasp an important material aspect of digital games, namely the control interface. Second, the role of control interfaces cannot be properly understood without analysing the interactive features of the digital game system and the embodiment of the player. I offer a conceptualisation of genre that takes these factors into account without giving up the virtues of existing accounts. I do this by both synthesising previous work and by introducing the term interaction modes to cover the embodied provinces of meaning entailed in digital game genres. To understand digital game genres and their development and appeal, we must look not only at the screen, but also at the coupling of interface and player and the resulting interaction modes. Third, I show how recent developments within the console gaming domain can be fruitfully analysed within this framework. In a brief conclusion, I summarise the results and outline a few perspectives for further work.

\section{Genre in literary theory and the cultural industries}

Their differences untold, contemporary genre theory overviews provided by individuals such as Rimmon-Kenan (2002), Frow (2006), Altman (1999) and Neale (2000) all argue for genre theories which focus less on stable taxonomies than on the fluid, situated and performative character of genres. Genre texts may relate to many categories at once and genres are seen as historically mutable and in constant development. Within film and television studies, the concept of genre has additionally been tied closely to institutional and industrial production methods. Film and television are thus seen as industrial enterprises which seek to minimise risk and maximise profits by using standardised production methods to 
make and market products that belong to specific genres or formulas (e.g., Altman, 1999; Feuer, 1992; Hesmondhalgh, 2007; Neale, 2000). The result is a play between continuity and change in genre developments: mostly the old and well-known, but with a sprinkling of the new and innovative, often by way of cross-breeding genres.

A related reason for such a genre concept is that it allegedly cuts across production, text and reception: genres and formats represent a "tacit contract" between producers and audience, and the product "honors that contract" (Feuer, 1992, p. 143). This minimises risk by "[suggesting] to audiences the kinds of pleasure they can attain by experiencing the product" (Hesmondhalgh, 2007, p. 23). Grodal (1997) focuses explicitly on the emotional pleasures of genre entertainment as well as on the role of human embodiment, the latter of which will be a major component of the following analysis. The following argument thus presupposes that audience expectations and embodiment play an important role in the appeal of entertainment, in general, and digital game entertainment, specifically. Before elaborating on this, I will introduce a genre theory which includes media technologies as a component in the genre analysis.

\section{Genre and media technologies}

Yates and Orlikowski (1992) define genre as "a typified communicative action invoked in response to a recurrent situation" (p. 301) ${ }^{1}$ and such genres are "characterized by similar substance and form" (Ibid.), where one aspect of form is the communication medium. The authors also, however, distinguish between "the physical means of communications (media) and the typified communicative action (genre)" (Yates \& Orlikowski, 1992, pp. 310-11), and taken together these formulations highlight a slight weakness in the consistency of the framework, viz. the tendency to conflate genre and medium. On the one hand, genres are defined partly by similarities of form, one subdimension of which is medium the medium is thus understood as part of the genre. On the other hand, media are to be distinguished from genres, the latter of which are understood as typified communicative actions. A reasonable reconstruction of the argument is that genre, once defined as typified actions, does not belong to specific media, but rather can move between media. Yates and Orlikowski themselves offer the following observation: "Though a genre's form may at one point include the medium, that genre may also expand into other media" (1992, p. 319). This quote hints at the kind of situation I want to substantiate here with regards to digital games. Some genres seem constituted by specific technologies, others less so. Such relations will change over time - and, importantly, technologies may also expand into other existing genres as part of such processes.

Later developments by the two authors introduce the related concepts of genre systems and genre repertoires, which cover mutually related genres and the "set of genres routinely enacted by a particular community"(Orlikowski \& Yates, 1994, p. 546). In the cultural industries, such genre systems can be identified at several levels, both at the level of industry 
sector, such as film, television, music and games, as well as within these sectors, where some major genres may include several subgenres.

I will later show how changes in digital game genre systems have included changes in situation, purpose, and material and formal aspects of digital game technologies accompanied by changes in player repertoires. Additionally, the tension between material media and genre should neither be disregarded nor subjected to exorcism. This is partly a matter of equally valid perspectives, since the same object may be analysed fruitfully with or without attention to material technology. My primary aim here is to contribute to the former of these perspectives, and the necessity of such a perspective will vary: I will argue that technology is integral to certain game genres while it can be seen as somewhat external to others. In other words, while some game genre definitions cut across aspects of technology, certain games - even if generic at some level - may be tied very closely to specific technologies.

In the following sections I will discuss interactivity as an important feature of digital games and argue that digital game genre theory, even when focusing on interactivity, still has a blind spot when it comes to the specific material aspects of interactivity.

\section{Digital games and interactivity}

Digital games, in this context, refer to arcade video games, home console video games, and games played on programmable personal computers; the terminology "computer and video games" is sometimes used to refer to all three domains at once, but I will use the shorter term "digital games" to refer to the superset of these categories. One of the most prominent debates within the fledgling community of digital game scholars focused not on their common digital nature, but on other ideas of shared lineage, namely whether digital games were narratives or games (the so-called narratology vs. ludology debate). Although it has become somewhat de rigueur to denounce this debate as both polemical and sterile, the discussion and its implications are still important. Discussions of overall and fundamental categorisation allow scholars to subsequently apply an established genre repertoire of academic theories and related analytical models to an empirical domain. Narratology and ludology both tend to abstract away from the technological basis of the medium and the present approach seeks to rectify these abstractions while preserving the contributions of both narratology and game studies. We can thus focus on interactivity, technology and embodiment and still acknowledge that digital games may share many traits with narratives and games.

It is a common assertion that digital technologies are interactive, and digital games are often referred to as interactive entertainment. ${ }^{2}$ Interactivity, however, is both deemed central to a whole field of studies (so-called new media) and almost invariably introduced as problematic, ill-defined, or not defined at all, as in, e.g., Heeter (2000), Jensen (1999) and Kiousis (2002). ${ }^{3}$ However, together with McMillan (2006), these writings provide several useful points and references for analysis. Kiousis (2002) argues that media interactivity can be investigated from three interrelated perspectives: the technology, the nature of the exchange 
and the users' perception. The focus here will be on technology. Heeter (1989) argues that interactive technologies can be analysed with respect to complexity of choice, user effort, responsiveness, monitoring of information use, ease of adding information and facilitation of interpersonal communication. It has been argued that the most important aspect of new media is their facilitation of mediated interpersonal communication (see Baym, 2010) and I will return to this point; but the majority of the discussion here will focus on the first four aspects as an analysis of user-to-system interactivity (McMillan, 2006): digital games may thus be individuated in terms of complexity, user effort, responsiveness and user monitoring. (Steuer, 1995) argues that certain kinds of interactive technologies are designed to foster a feeling of telepresence, a sense of "being there". He also distinguishes between vividness and interactivity, where both are relevant here. Vividness depends on sensory breadth and depth, whereas interactivity depends upon speed, range of choices and mapping of user actions. Both vividness and interactivity influence the experience of telepresence. Additionally, Steuer points out that interaction should be seen as meaningful from the perspective of the interactor, a point central to the work of both Laurel (1993) and later Salen and Zimmerman (2004) in their analyses of game interaction.

A key point mostly ignored or left implicit in some of the work on interactive technologies is that all of the aforementioned aspects of interactive technologies are interrelated, and together describe not just dimensions of media but rather media relationships - all of the dimensions have ties to the embodied skills of the user and the way the system reacts to the exercise of those skills. Gaming is an exercise in skills and attainment of goals, and some of the genre theories related specifically to digital games thus tie genre to purpose and goals.

\section{Digital game genres: Purpose and goals}

Wolf's (2001) proposal for a digital game genre framework is premised on the types of interactivity demanded by the game structure, i.e., what the player is supposed to do to succeed. The result is a set of categories more detailed and sometimes quite different from genres used by the game industry, game press and popular websites, ${ }^{4}$ but it includes most of these major game genres such as "sports", "adventure", "platform", "combat", "racing", "strategy" and "shooter" etc., and offers many examples. A related but more recent example of digital game genre analysis is offered by Egenfeldt-Nielsen, Smith, and Tosca (2008), who also base game genres on "a game's criteria for success" (p. 41) resulting in four overall genres, namely Action, Adventure, Strategy and Process-oriented games. This genre system abstracts away from differences of material and digital technology and is based squarely on purpose, understood as the success criteria inherent in the design structure. The system does not include subgenres such as those used by the industry press, e.g., platform or racing games.

When combined, these two perspectives allow for a reasonably fine-grained and crosscutting analysis of established game genres and formulas; e.g., a combat or racing game may 
veer towards action or strategy. The identification of the particular kinds of interactivity seen in games is also compatible with the more general features of interactive technologies outlined above, e.g., action and strategy imply different kinds of complexity and cognitive effort to achieve success, and the game system monitors and reacts to player actions in different ways. In addition, such a genre framework allows for combination with established genre categories such as western, sci-fi or fantasy, all of which are relevant for classifying products within the major game genres of action, adventure and strategy. What is missing, however, is a theorisation of how material technology enters this mix of interactivity, overall success criteria and established genres. My argument is that material interfaces can play a substantial role in game genre developments: specifically, the innovation, evolution and consolidation of interface technologies and their connections to game genres have been a key distinguishing feature of the recent motion game phenomenon - but interface evolution is also a strong undercurrent which runs through the history of digital games.

In the following sections of the article, I will bring together the perspectives of the previous sections. First, I will show how these can be used to analyse the history and continued evolution of digital games and the material technology involved. Second, I will augment the framework further by showing how material technology and game interactivity may be tied to player embodiment.

\section{Arcade and home: Situation and technology}

Newman's (2004) discussion of arcade games and home platforms identifies location and context as relevant parameters in digital game analysis and this can be elaborated with the components of genre already laid out initially, namely purpose, social situation and the material structure of technology. Even if they ostensibly involve the same game title and the same genre, such as platform or racing, arcade and home console games may be differentiated by these factors. Arcade versions thus have an overall and generally acknowledged purpose of continual payment of small amounts of money to gain access to playing games in a short amount of time in a public venue with a possibility for spectators. In the case of consoles, one pays a comparatively larger sum of money once to buy hardware and games for private ownership and subsequent unlimited consumption in the home, with a resulting audience restricted to the private sphere. The latter crucially involves buying both console hardware and individual games.

The home console market is currently divided by three hardware companies, i.e., Microsoft, Sony and Nintendo, all of which also publish game software for their own console systems. This market has the structure of an oligopoly and sees widespread use of series, formulas and sequels to minimise risk (for overviews, see Egenfeldt-Nielsen et. al, 2008; Newman, 2004). All console platforms structure the situation of home gaming in particular ways since once consumers have committed to a specific home system, they need to buy the game products for that particular system as there is no interoperability, ${ }^{5}$ i.e., Nintendo 
games can only be played on Nintendo hardware etc. This means that every console has a genre system of its own, including flagship exclusive titles and small differences in lineup and emphasis. ${ }^{6}$ This also, crucially, means that interface devices included in the system setup may be proprietary as well.

\section{Material media: Interface and control}

Interface devices are the parts of media technologies that allow human users to exchange information with media systems. In most digital games, physical actions of users are mapped (Norman, 2002; Steuer, 1995) to audiovisual representations: players manipulate the interfaces to jump, shoot or drive etc. in the world of the game. Newman points to the differences found in "custom cabinets and interface technologies" (2002, p. 14) which have been an important part of arcade history (Kent, 2001). In Newman's words, this is a difference in the "level of physicality" of interfaces, where arcade games have traditionally offered interface technologies that are comparatively more complex and involving than those seen in home consoles, which have often used standardised controllers.' It is left implicit in Newman's argument that such complex interfaces very often model real world material tools or technologies such as weapons and/or vehicles; the history of arcade machines is replete with guns, submarine periscopes, airplane cockpits and race car interiors. All of these arcade games can be said to simulate specific real-world scenarios of technologically assisted action. They are thus a mixture of digital and material simulation technologies and this mix of digital and material runs through the history of all interactive entertainment up to present developments.

All game interfaces are closely tailored to human factors, i.e., physical embodiment - just like any other technology which is intended for human interaction. As such, it is fundamentally and importantly correct when Newman says that video games are "a form of embodied experience" (Newman, 2002, quoted in Newman, 2004, p. 27), but it should additionally be asserted that all human experience is fundamentally embodied. Not only does our common physical embodiment influence the ways we think (see Gallagher 2005; Lakoff, 1987; Lakoff \& Johnson, 1980), but we experience the world as acting physical bodies. Material interfaces, their affordances for physical actions, and the mappings from physical action to representation are usually given short shrift in genre analyses. But, the combination of our common physical embodiment and generic physical interfaces leads to generic structures of action, or what I call interaction modes.

\section{Interaction modes: The structured coupling of player and system}

Frow (2006) argues that genres presuppose and invoke implicit knowledge which is organised schematically, a point congruent with other cognitive approaches to narrative and genre (e.g., Bordwell, 1985; Grodal, 1997). This has obvious parallels with the tacit contract 
mentioned previously: genre audiences know what to expect from genre products, in terms of form, content and expected gratifications. Frow refers to Alfred Schutz' concept of "finite provinces of meaning" to describe the overall set of such genre-specific schemas, but he also acknowledges that "Schutz defines these provinces as experiential rather than representational" (2006, p. 87). ${ }^{8}$ A shift in focus from mental representation to active embodied experience enables us to restate genres as not only representational provinces of meaning, but, in addition, embodied provinces of meaning as action. Genre not only implies structured knowledge, but structures of embodied action: we know genres and we know what to do with genres.

A given digital game genre can be seen as such a province of meaning as action. Following this, the interaction mode identifies generic structures of physical activity when interacting with the total game system. Players need to move their bodies in specific ways to affect the game system and interaction modes are thus integral to defining games as finite provinces of embodied interaction. It follows that an interaction mode is, emphatically, not the same as a "game genre", as that concept is described above, since it does not pick out genericity at the level of "racing", "action", "real-time strategy" or "science-fiction", but rather adds a necessary dimension of embodied experience to such generic descriptions.

It is possible to further integrate Orlikowski and Yates' idea of genre repertoire with interaction modes since the overall set of embodied skills for interacting with technology is a crucial part of the genre repertoire of agents in a given community: gamers know their games, genres and the requisite success criteria, and they also know specific interfaces and the requisite interaction modes.

On the one hand, game genres as defined above allow for cutting across technology by picking out categories such as racing games and shooters across consoles, arcade and PC platforms. So, even if individual games are not cross-platform, they will often participate in cross-platform genres. On the other hand, interface devices and their requisite interaction modes may be very important to the appeal of certain products. Such interfaces may be specific and/or proprietary to both arcade versions and console systems in various combinations, a tendency that runs through digital game history. Early targeting games available both in arcade and home versions used light guns pointed at the display; this genre was more or less defined by the combination of the physical gun accessory, its particular sensing technology, and the resulting affordances for a specific interaction mode, namely aiming. An arcade game like After Burner, a combat aircraft action game, was ported to home consoles, but the arcade version included a large cockpit with hydraulics. ${ }^{9}$ The more recent music instrument interfaces, pioneered by the Beatmania arcade games, saw a period of brief but huge popularity in their home versions, i.e., the Guitar Hero and Rock Band franchises. ${ }^{10}$ And interfaces and interaction modes may be so idiosyncratic that they challenge genericity outright: a Nintendo console game such as Donkey Conga is obviously a rhythm game, but at the same time one of a kind - it comes with a pair of actual congas serving as interface. 
Motion gaming has developed within this space of the generic and the specific with regard to interfaces and interaction modes: motion games are not just designed for a specific console platform, but are designed for a specific input device and thus come with particular interaction modes implied. The next sections describe how the recent motion gaming developments within the home console system domain involve changes in technology and implied interaction modes and thus in genre systems and player repertoires. I will start by introducing the console that put motion gaming on the game map: the Nintendo Wii.

\section{Gaming in motion: The Wii}

The Nintendo Wii console was launched in 2006 as a bundled package of console hardware, the new Wiimote controller and software designed specifically for this new control interface. This ensured that the video game system functioned as an integrated configuration of compatible artefacts as is customary with all consoles. But this gaming system implied changes in the overall recurrent situation of playing video games in a private setting. The Wii and especially its Wiimote are structured for, but also structures, certain embodied uses of the system, i.e., it is a component in interaction mode developments and thus in genre developments. People had to familiarise themselves with this new interaction mode and its specific demands, such as upright posture and high-intensity wider reaching movements. The generic function of the Wiimote is that of controller, ${ }^{11}$ but at launch time this instantiation was highly novel. At the same time it was to serve as a generic input device from thereon. I will now look at how this mix of the specific and the general - the idiosyncratic and the regularly occurring - had an impact on gaming situation, interaction genres, traditional game genres and their development. Or, to put it differently: in what respects did the Wii and its Wiimote controller make a difference in existing genres?

The computational power and structure of the Wii console itself did not change much when compared to previous generations of consoles including its own immediate predecessor, Nintendo's Gamecube. Nintendo did not follow the lead of rivals Sony and Microsoft which were both committed to the development of system architectures that allowed for an increase in computational power and, as a result, the output of high definition audiovisual content. In Steuer's terms, Microsoft and Sony had opted for an increase in depth and breadth of the video and audio signals, a console launch strategy that had become well entrenched at that time (see Newman, 2004). They also pursued a hardware convergence strategy, especially Sony, whose Playstation 3 was marketed on its multimedia capabilities and Blu-ray disc playback. ${ }^{12}$

Nintendo, in contrast, innovated on the level of input technology in a move that deftly targeted a blind spot in the then-current video game system setups and their affordances. Instead of going for increased immersion and/or telepresence afforded by increases in computational power and audiovisual spectacle, Nintendo (arguably) sought to create both increases and differences in kinds of immersion by reconfiguring the relationship between the 
user actions and the actions represented on-screen; the most important aspect was increased isomorphism between the two (see Gregersen \& Grodal, 2009). What you did, the avatar on screen did. This strategy more or less upended the console market and possibly expanded it.

The Wii thus introduced a new configuration of game technology which allowed the computer game system to register user activity and respond to this in a different way than previous consoles. But, this also meant that the novel Wiimote had to be coupled to game software that utilised the new controller in a meaningful way. The established overall genre systems of the previous console generations was thus subjected to potential changes less pressing and nudging, more actual swinging, stabbing and turning. Reviews of several games pointed towards problems with integration of the new technology, but Nintendo had made sure that the most rudimentary of genre systems was in place at launch: buying a Wii entailed the simultaneous acquisition of console hardware, the new controller and the game software Wii Sports.

\section{Motion gaming and established game genres}

With regards to established game genres understood as previously presented, Wii Sports included four fairly primitive action sports games, i.e., Wii Tennis, Wii Boxing, Wii Baseball and Wii Bowling. The Wiimote and Wii Sports thus exemplify how specific aspects of technology may be said to serve a constant function, namely that of input. The particular nature of this input technology, however, enabled genre developments within the established genre of action sports games in terms of overall purpose and thematic content and structure. If one shifts the perspective and prioritises the actions of the embodied player, i.e., the interaction mode, the Wiimote provided a major development of both the sports genre and, potentially, the overall genre of action: players now got to experience higher intensities in their body movements and the audiovisual feedback provided a richer isomorphism between embodied and represented actions with potential - but certainly not guaranteed - effects on the experience of agency and virtual body ownership. ${ }^{13}$

These physical movements were all taken from prototypical activities known from everyday mediated life. A key component of the new interaction modes and the resulting genre repertoire of players, namely its motor activity structures, was thus recruited from a repertoire of previously existing - but not necessarily game-related - motor activities: the games were, above all, simple motor action simulations.

Another related development was Nintendo's establishments of stylised, customisable avatars - called Miis - which were cross-game entities. As such, increased ownership of the represented virtual bodies was technologically scaffolded by allowing users to familiarise themselves with a partly customised and consistent virtual self-presentation across the consoles' genre system. An additional and important part of these genre developments was a revision of a particular idea about games, namely that they should be challenging and involve special skills: Wii Sports was, initially at least, trivially easy. This follows an overall 
trend in digital games towards so-called casual gaming, i.e., games which do not demand big investments in terms of time or skills. But the "ease of access"-aspect of casual gaming cuts across several genres and platforms, and while casual gaming was certainly part of Nintendo's strategy, the Wiimote's specific impact was due to the fact that players now could (and in many instances had to) perform physical actions in front of the screen (for discussion see also Juhl, 2010).

This combination of novel interface, avatar integration and casualness ran counter to dominant and previously successful strategies employed by Microsoft and Sony in the overall console domain. Key games related to those platforms had traditionally been the quasirealist audiovisual action spectacle of games series such as Gran Turismo, Grand Theft Auto and Halo. Nintendo took a different route by launching their new technology with a small suite of games which were very simple in terms of both representation and the actions required in order to meet the challenge - and the total genre system formed around the recurrent situation of home gaming changed slightly as a result. All of the factors mentioned above were to play some role in the responses of Sony and Microsoft to Nintendo's market grab. The innovative nature of the Wiimote and its dominant interaction mode of swinging and hitting would gradually be replaced by a larger class of related interaction modes identified at a higher level of abstraction: the overall category of motion gaming.

At this point, however, one might ask whether motion gaming is a genre in itself. This is part of a well-known and thorny issue in genre studies, namely where the concept genre is properly applied in terms of hierarchy and level of abstraction (see Miller, 1984, and Yates \& Orlikowski, 1992, for some of the positions available). I find a pragmatic and processual standpoint as advocated by Yates and Orlikowski most useful: questions of genre and subgenres cannot be settled once and for all, but rather allow us to pose interesting questions in particular contexts at specific points in time. I do not see motion gaming as a genre in and of itself, but rather as a phenomenon that ties in with genre developments, since physical embodiment, interfaces and the resulting interaction modes have had and still have important connections to the evolution of game genres.

\section{Motion gaming developments: Genre diversification, convergence and interpersonal communication}

Microsoft and Sony soon followed Nintendo's lead by developing and launching their own motion control devices. Nintendo had for their part continued with their enlarging of the game console market with their fitness-oriented Wii Fit balance board, but both in order to preempt attacks within the more traditional gaming audience and to follow up their previous hit, they released Wii Sports Resort in 2009. This game came with a Wii Motion Plus hardware add-on for the original Wiimote, which was marketed as a more precise and responsive motion input controller. In both cases the combination of hardware and software was used to sell a complete experience, but in the latter instance, the category and 
success of previous motion gaming technology, its tailored game software, and the previous emphasis on social game situations was invoked by Nintendo itself on the box:

The follow-up to Wii Sports invites you to challenge friends and family in a host of new games like Archery, Basketball and Swordplay, and then take to the ocean around the island to enjoy fun filled water sports. Take your Wii Remote to the next level and discover all-new ways to play - with Wii motion plus (Nintendo, 2009).

Sony's Move controller from 2010 combined Wii Remote-like motion sensors and a standard web camera (the Playstation Eye), the latter of which has a precursor in Sony's own Eye Toy camera for Playstation 2, which allowed users to control on-screen actions without manipulating a physical controller. Sony's launch shows how many different genres of games may be crossbred with motion technology - e.g., sports, light gun-like targeting and casual party games - but a typification of recurrent action had already established itself to the extent that the act of launching a motion gaming control device should include a suite of sports games tailored to the interface. Sony's offering in this domain, called Sports Champions, tried to leverage Sony's position as a deliverer of non-casual, complex and challenging gaming experiences as evidenced from their box blurb:

\begin{abstract}
Compete in a series of ultra-realistic events that demand accuracy, ability and skill - just like real sport. Your movement is the key to success in each event; so just how good can you get? Perfect real life sporting techniques - grip your bow, reach for an arrow, take aim, pull back and let fly. Use one or two Playstation Move motion controllers to play Beach Volleyball, Disc Golf, Gladiator Duel, Archery, Table tennis and Bocce. Challenge your friends to multiplayer matches and work up a sweat to prove you're the greatest champion of all. See your winning scores on the online leaderboards and upload victory poses to Facebook (Sony, 2010).
\end{abstract}

The range of sports offered here is tied to both the proprietary controller and to social antagonism and the exercise of skills. Also worth noting is the use of "ultra-realistic": such discourse of realist immediacy (Bolter \& Grusin, 2000) is employed extensively by the gaming industry, and in this case it is applied to mundane and low-intensity sports, such as Bocce, as well as the anachronistic, fictionalised, and high-intensity combat of Gladiator Duel. Both games feature isomorphic motor control, but are otherwise very different in terms of both semantic content and player success criteria, i.e., domestic low-key leisure vs. fantastic and brute physical antagonism. Note also the direct ties from the agonistic structure to Sony's own proprietary SNS-like Playstation Network (friends and leaderboards) and the integration with the dominating third-party player in the area of SNS, i.e., Facebook. One chief difference from Microsoft and Nintendo, however, is the lack of cross-game customised avatars.

Microsoft's Kinect technology from 2010 was marketed with the tagline "You are the controller". In contrast to the other two offerings (but just like the original Eyetoy, only 
more complex), the Kinect is a camera-based interface which features no physical controller parts. The bundled game Kinect Adventures combine racing genres and primitive sports genres with possibilities for sharing pictures and self-created motion capture animation of "living sculptures". Kinect Sports delivers the obligatory sports package complete with avatar integration, and one of the most successful launch games was Dance Central, a dance game that arguably allowed for the most complex player gesture registration seen in digital games to date. ${ }^{14}$

The Kinect sensor technology also shows how certain combinations of material and digital media may interact with genre not just understood as game genres, but also communication genres, as described by Orlikowski and Yates. The games using person-to-avatar gesture mapping allow for in-game expressivity, but the recently launched ${ }^{15}$ product, Avatar Kinect, aims to hybridise game and communication genres even further. Avatar Kinect uses the motion capture capabilities of the Kinect sensor to facilitate interpersonal communication in the form of chat between avatars with facial animations which are mapped from the user's real facial musculature to stylised animated avatar behaviour. This is an instance of synchronous group communication (in one-to-one and many-to-many forms), well known from computer-mediated chat applications, a sizable niche of which is avatar-based (see, e.g., Taylor, 2002). Avatar Kinect seeks to augment this experience with an increase in breadth and depth of interpersonal communication cues. It also forges yet another link to the dimension of self-presentation inherent in the Xbox live avatar system, which also means that the added dimension of body isomorphism coupled to interpersonal communication may in turn foster even greater ownership of the avatar self-representation across situations.

\section{Conclusion}

I hope to have shown that technology has played an important role in digital game genre developments and that the concept of interaction modes is a helpful addition to existing game genre theory frameworks, especially in the context of technologies such as the Wiimote controller and the Kinect sensor. One outstanding issue is the question of compatibility and similarity between different interaction modes and resulting genre developments. Schutz' original proposal defined the embodied provinces of meaning as finite and thus to some extent incompatible with each other; they mark off different ways of engaging reality, so to speak. This point can still be acknowledged while advocating a softer version of the thesis: there is indeed something finite and identifiable in these control technologies and their interaction modes, but at the same time there are compatibilities across genres when defined at other levels, as just shown with the sports genre and dance genres. In addition, an action shooter such as Killzone 3 can be played with a standard controller or with a rifle-shaped Move accessory - the same game software thus comes with two different interaction modes. 
A final and particularly important question is how these motion control interfaces and related developments within digital games may impact the larger field of mediated communication. Each gaming console system has by now integrated applications associated with important genres from computer-mediated communication: all three gaming platforms now include features well known from social network sites, such as "friends" and messaging systems, and the potential impact of control devices must thus be understood both in relation to the gaming console system but also to communication platforms and genres which are not exclusive to games. In this regard, the Kinect controller especially merits sustained attention in the coming years. Although its early game uses saw a kind of 'soft technological determinism' (Pool, 1983) in its limited uses as a game controller, Microsoft's strategy with regards to availability of Kinect Software Development Kits together with the innovations of a Kinect-centred PC hacking community (see kinecthacks.net for some impressive examples) have already demonstrated how the combination of industrial strategy, off-theshelf technology and enthusiast users may lead to genre developments completely outside the realm of original market orientation: the Kinect, or similar technologies, may become a component in many kinds of interactivity, both user-to-system and user-to-user (see McMillan, 2006).

In summary, genre theory should be able to take on board the concept of interaction modes to identify salient patterns of generic physical interaction as an important part of genre understood as embodied provinces of meaning. Technology and the connections to our physical embodiment can be key components in both game and communication genre developments. Thus, genres may move and cross-breed across media technologies, but technologies and embodiment may also matter in genre. Genres evolve, and so should genre theory.

\section{Acknowledgements}

Support for this research was provided by the Carlsberg Foundation. The author would like to thank Jason Gendler, Thomas Wiben Jensen, Rasmus Kjærgaard Rasmussen, the editors and the anonymous reviewers for helpful comments on earlier drafts of this article.

\section{Notes}

1. The theory is an elaboration of Carolyn Miller's (1984) seminal work on rhetorical genres.

2. See Newman (2004, p. 7) for the cultural implications of this and other related labels.

3. Jensen (1999) argues for a distinction between person-to-person interaction and interactivity understood as a person interacting with technology. While this distinction is important, there is little consensus on restricting interactivity to only the latter - McMillan (2006), for instance, distinguishes 
between user-to-user and user-to-system interactivity; also, both of these can manifest themselves in the same situation.

4. See also Newman (2004) for an overview of industry game genres.

5. All console manufacturers have exploited the strategy of securing console exclusive games (see, Egenfeldt-Nielsen et al., 2008, p. 90). Even when users turn to software piracy, they are still locked into that particular system of technology.

6. For instance, Gran Turismo (Sony) vs. Forza Motorsport (Microsoft) and Killzone (Sony) vs. Halo (Microsoft).

7. So have arcade games, the most widespread being a setup of one joystick and a set of buttons.

8. Schutz is a major inspiration to Miller, who subsequently grounds the work of Yates and Orlikowski, and Frow.

9. After Burner was available in both cockpit and non-cockpit arcade versions, see http://www.arcademuseum.com/game_detail.php?game_id=6821. Accessed September 21, 2011.

10. Rock Band 3 is compatible with actual MIDI drums as interface - a drumming game with an ultrarealistic interaction mode.

11. Including its specific sensor technology, which is excluded here in the interest of brevity.

12. Previous console launches promising increases in such factors had to some extent become generic, a set of typified actions in response to recurrent situations on the level of corporate strategy. See Dovey and Kennedy (2006) on "upgrade culture".

13. See Gregersen and Grodal (2009) for more on action isomorphism, agency, and ownership of bodies and actions.

14. The more difficult levels in Dance Central show that motion gaming should certainly not be directly equated with the absence of challenge.

15. Fall 2011.

\section{References}

Altman, R. (1999). Film/Genre. London: BFI.

Baym, N.K. (2010). Personal Connections in the Digital Age. Cambridge, UK: Polity.

Bolter, J.D. \& Grusin, R. (2000). Remediation. Understanding New Media. Cambridge, MA: MIT Press.

Bordwell, D. (1985). Narration in the Fiction Film. Madison: University of Wisconsin Press.

Dovey, J. \& Kennedy, H.W. (2006). Game Cultures. Computer Games as New Media. Maidenhead: Open University Press.

Egenfeldt-Nielsen, S, Smith, J.H. \& Tosca, S.P. (2008). Understanding Video Games. The Essential Introduction. New York: Routledge.

Feuer, J. (1992). Genre Study and Television. In R.C. Allen (Ed.), Channels of Discourse, reassembled. Television and contemporary criticism. Second Edition. Routledge): London.

Frow, J. (2006). Genre. London: Routledge.

Gallagher, S. (2005). How the body shapes the mind. Oxford: Clarendon.

Gregersen, A.L., \& Grodal, T. (2009). Embodiment and Interface. In B. Perron \& M.J.P. Wolf (Eds.), The Video Game Theory Reader 2. London: Routledge.

Grodal, T. (1997). Moving Pictures. A New Theory of Film Genres, Feelings and Cognition. Oxford: Clarendon Press.

Heeter, C. (1989). Implications of New Interactive Technologies for Conceptualizing Communications. In J.L. Salvaggio \& B. Jennings (Eds.), Media Use in the Information Age: Emerging Patterns of Adoption and Consumer Use (pp. 217-236). Hillsdale, NJ: Lawrence Erlbaum Associates. 
Heeter, C. (2000). Interactivity in the Context of Designed Experiences. Journal of Interactive Advertising,

1(1). Retrieved from http://www.jiad.org/article2

Hesmondhalgh, D. (2007). The Cultural Industries. 2nd Edition. London: Sage.

Jensen, J.F. (1999). 'Interactivity' - Tracking a New Concept in Media and Communication Studies. In P.A.

Mayer (Ed.), Computer Media and Communication (pp. 160-187). Oxford: Oxford University Press.

Juul, J. (2010). A casual revolution. Reinventing video games and their players. Cambridge, Mass.: MIT Press.

Kent, S.L. (2001). The Ultimate History of Video Games. New York: Three Rivers Press.

Kiousis, S. (2002). Interactivity: a concept explication. New Media Society, 4(3), 355-383. doi: $10.1177 / 146144480200400303$

Lakoff, G. (1987). Women, Fire, and Dangerous Things. What Categories Reveal about the Mind. Chicago: University of Chicago Press.

Lakoff, G. \& Johnson, M. (1980). Metaphors We Live By. Chicago: University of Chicago Press.

Laurel, B. (1993). Computers as Theatre. Reading, Mass.: Addison-Wesley.

McMillan, S.J. (2006). Exploring models of interactivity from multiple research traditions: Users, documents, and systems. In L.A. Lievrouw \& S. Livingstone (Eds.), The Handbook of New Media. Updated Student Edition (pp. 205-229). London: Sage.

Miller, C.R. (1984). Genre as social action. Quarterly Journal of Speech, 70(2), 151-167.

Neale, S. (2000). Genre and Hollywood. London: Routledge.

Newman, J. (2004). Videogames. London: Routledge.

Norman, D.A. (2002). The Design of Everyday Things. New York: Basic Books.

Orlikowski, W.J., \& Yates, J. (1994). Genre Repertoire: The Structuring of Communicative Practices in Organizations. Administrative Science Quarterly, 39(4), 541-574.

Pool, I. de Sola. (1983). Technologies of Freedom. Cambridge, MA: The Belknap Press of Harvard University Press.

Rimmon-Kenan, S. (2002). Narrative Fiction. 2. ed. London: Routledge.

Salen, K. \& Zimmerman, E. (2004). Rules of Play. Game Design Fundamentals. Cambridge, MA: MIT Press.

Schutz, A. (1962). Collected papers I. The Problem of Social Reality. The Hague, NL: Martinus Nijhoff.

Steuer, J. (1995). Defining virtual reality: dimensions determining telepresence. In F. Biocca \& M.R. Levy (Eds.),

Communication in the age of virtual reality (pp. 33-56). Hillsdale, NJ: Lawrence Erlbaum Associates.

Taylor, T.L. (2002). Living digitally: Embodiment in Virtual Worlds. In R. Shroeder (Ed.), The Social life of Avatars. Presence and Interaction in Shared Virtual Environments. London: Springer-Verlag.

Winograd, T. \& Flores, F. (1986). Understanding Computers and Cognition: A New Foundation for Design.

Norwood, NJ: Ablex Publishing Corporation.

Wolf, M.J.P. (Ed.). (2001). The Medium of the Video Game. Austin: University of Texas Press.

Yates, J. \& Orlikowski, W.J. (1992). Genres of Organizational Communication: A Structurational Approach to Studying Communication and Media. The Academy of Management Review, 17(2), 299-326.

Andreas Gregersen
Assistant Professor, PhD

Film and Media Studies Section,

Department of Media, Cognition and Communication

University of Copenhagen, Denmark

agr@hum.ku.dk 